\title{
Emerging role of microRNAs in modulating endothelin-1 expression in gastric cancer
}

\author{
KUO-WANG TSAI ${ }^{1 *}$, LING-YUEH HU ${ }^{2 *}$, TING-WEN CHEN ${ }^{3,4}$, SUNG-CHOU LI $^{5}$, MENG-RU HO ${ }^{6}$, \\ SHOU-YU YU ${ }^{1}$, YA-TING TU ${ }^{1}$, WEI-SHONE $\mathrm{CHEN}^{7}$ and HING-CHUNG LAM ${ }^{8}$ \\ ${ }^{1}$ Department of Medical Education and Research, Kaohsiung Veterans General Hospital, Kaohsiung; \\ ${ }^{2}$ Institute of Biomedical Sciences, Academia Sinica, Taipei; ${ }^{3}$ Molecular Medicine Research Center, Chang Gung University, \\ Taoyuan; ${ }^{4}$ Bioinformatics Center, Chang Gung University, Taoyuan; ${ }^{5}$ Genomics and Proteomics Core Laboratory, \\ Department of Medical Research, Kaohsiung Chang Gung Memorial Hospital and Chang Gung University \\ College of Medicine, Kaohsiung; ${ }^{6}$ Biodiversity Research Center, Academia Sinica, Taipei; \\ ${ }^{7}$ Department of Surgery, Veterans General Hospital, Taipei; ${ }^{8}$ Center for Geriatrics and Gerontology, \\ Kaohsiung Veterans General Hospital, Kaohsiung, Taiwan, R.O.C.
}

Received July 24, 2014; Accepted October 22, 2014

DOI: $10.3892 /$ or.2014.3598

\begin{abstract}
Endothelin-1 (ET-1) is a small 21-amino acid peptide that is known to exert diverse biological effects on a wide variety of tissues and cell types through its own receptors. The ET-1-ETRA axis is frequently dysfunctional in numerous types of carcinomas, and contributes to the promotion of cell growth and migration. microRNAs (miRNAs) are small non-coding RNAs that play a critical role in carcinogenesis through mRNA degradation or the translational inhibition of cancer-associated protein-coding genes. However, the role of ET-1 and the relationship between ET-1 and miRNAs in gastric cancer remain unknown. Results of the analysis of the database of The Cancer Genome Atlas (TCGA) revealed that ET-1 is significantly overexpressed in gastric cancer cells when compared with its expression in adjacent normal cells. Exogenous ET-1 significantly enhanced gastric cancer cell proliferation, implying that ET-1 plays an oncogenic role in gastric cancer carcinogenesis. Using a luciferase reporter assay we showed that 18 miRNA candidates had a significant silencing effect on ET-1 expression by up to $20 \%$ in HEK293T cells. Among them, 5 miRNAs (miR-1, miR-101, miR-125A, miR-144 and let-7c) were shown to be involved in ET-1 silencing through post-transcriptional modulation in gastric cancer. Our data also revealed that DNA
\end{abstract}

Correspondence to: Dr Kuo-Wang Tsai, Department of Medical Education and Research, Kaohsiung Veterans General Hospital, Kaohsiung 813, Taiwan, R.O.C.

E-mail:kwtsai@vghks.gov.tw; kwtsai6733@gmail.com

Dr Hing-Chung Lam, Center for Geriatrics and Gerontology, Kaohsiung Veterans General Hospital, Kaohsiung, 813, Taiwan, R.O.C. E-mail: hclam@vghks.gov.tw

*Contributed equally

Key words: microRNA, endothelin-1, gastric cancer, miR-1 hypermethylation contributes to the silenced miR-1 expression in gastric cancer cells. The ectopic expression of miR-1 significantly inhibited AGS cell proliferation by suppressing ET-1 expression. Overall, our study revealed that ET-1 overexpression may be due to DNA hypermethylation resulting in the silencing of miR-1 expression in gastric cancer cells. In addition, we identified several miRNAs as potential modulators for ET-1 in gastric cancer, which may be used as targets for gastric cancer therapy.

\section{Introduction}

Endothelin (ET) was first identified as a vasoconstrictive 21-amino acid S-peptide derived from vascular endothelial cells. It is currently known to exert diverse biological effects on a wide variety of tissues and cell types through its own receptors $(1,2)$. Three ETs (ET-1, ET-2 and ET-3), 2 G-proteincoupled receptors (ETRA and ETRB) and 2 ET-converting enzymes (ECE-1 and ECE-2) comprise the ET axis (2). The ET-1 gene contains 5 exons located on chromosome 6 , and expresses a 212-amino acid precursor, preproendothelin-1 (PPET-1). PPET-1 is converted by protease to form an intermediate peptide consisting of 38 amino acids, big-ET-1, which is further cleaved by ECE-1 or ECE- 2 to release the mature form of ET-1 (21 amino acids) $(3,4)$.

An increase in ET-1 expression has been detected in various malignancies including lung, prostate, colorectal, liver, breast and ovarian cancers, as well as melanomas, and may contribute to cell growth, metastasis and angiogenesis, and suppression of apoptosis (4-11). ET-1 typically induces proliferation through the ETRA receptor, and decreased ETRB expression has frequently been implicated in the pathogenesis of esophageal squamous cell carcinoma and nasopharyngeal carcinoma, as well as prostate and ovarian cancers (12). The promoter regulatory region of the ETRB gene contains a 5' $\mathrm{CpG}$ island which is commonly (70\%) methylated, suggesting that ETRB downregulation occurs through DNA hypermethylation in human 
carcinomas (13-17). In gastric cancer, a high level of ET-1 has been detected in plasma, which may be a reliable marker for predicting recurrence $(18,19)$. Stow et al $(20)$ summarized previous studies and revealed that ET-1 transcription can be enhanced using numerous physical and chemical stimuli such as phorbol esters, thrombin, angiotensin II and insulin, by activating signaling transduction pathways including the protein kinase $\mathrm{C}(\mathrm{PKC})$ and JUN-terminal kinase (JNK) pathways. In addition, GATA family transcription factors, $\beta$-catenin, transforming growth factor $\beta$ (TGF $\beta$ ) and hypoxia-inducible factor-1 (HIF-1) can enhance ET-1 transcriptional activity by modulating its promoter $(8,12)$. Therefore, unbalanced stimulators and impaired signaling that frequently lead to ET-1 overexpression trigger the activation of the ET axis, and promote cancer cell progression through autocrine and paracrine signaling. However, the detailed functions and regulatory mechanisms of ET-1 in gastric cancer remain unknown. The number of studies has recently been increasing regarding small non-coding short RNA microRNAs (miRNAs) in cancer development through post-transcriptional regulation of the expression of oncogenes or tumor-suppressor genes. miRNAs are deregulated in numerous disease states, particularly in cancer, making them novel molecular indicators that are critical for early diagnosis, prognosis, and therapy. Previous studies have demonstrated several instances of abnormal miRNA expression in gastric cancer cells compared with adjacent normal cells $(21,22)$. These dysfunctional miRNAs may disrupt the balance of their target genes, resulting in the downregulation of tumor-suppressor genes and oncogene overexpression in gastric cancer.

Previous studies have revealed that miRNAs play a critical role in modulating ET-1 expression by targeting the 3'untranslated region (3'UTR), including miR-1, miR-125a, miR-125b, miR-155 and miR-199 (23-28). To date, the biological function and regulating mechanisms of ET-1 by miRNAs in gastric cancer remain unknown. In the present study, we demonstrated that ET-1 plays an oncogenic role in gastric cancer growth, and identified several potential target sites for miRNA candidates harbored within the 3'UTR of ET-1 using a bioinformatics approach. We identified 5 dysfunctional miRNAs that may contribute to ET-1 overexpression in gastric cancer, to provide a potential approach and novel targets for therapy.

\section{Materials and methods}

Cell line and nuclear acid extraction. Five human gastric cancer cell lines, AGS, AZ-521, HR, NUGC and TSGH, were obtained from the American Type Culture Collection, and maintained in Dulbecco's modified Eagle's medium (DMEM) supplemented with $10 \%$ inactivated fetal bovine serum (FBS; Invitrogen, Carlsbad, CA, USA). After 5-Aza-dC $(10 \mu \mathrm{M})$ treatment for 4 days, RNA was extracted using TRIzol reagent (Invitrogen), according to the manufacturer's protocol. After RNA extraction, the interface was subjected to DNA extraction with a back extraction buffer (4 M guanidine thiocyanate, $50 \mathrm{mM}$ sodium citrate and $1 \mathrm{M}$ Tris). Genomic DNA was then precipitated with $\mathrm{EtOH}$.

Constructed reporter of ET-1 and the miRNA expression vector. ET-1 3'UTR was amplified from AGS cDNA by using the specific primer. The amplified ET-1 3'UTR was cloned into the pmiR-RB-Report vector, between the XhoI and Not I restriction sites. The primary miRNA transcripts were amplified from the genomic DNA of AGS cells by using miR-specific forward and reverse primers. The amplified PCR products were then cloned into the $\mathrm{pLKO}$ vector, between the AgeI and EcoRI restriction sites. All constructs were further confirmed through direct sequencing. The individual primers used in the present study are shown in Table I.

Luciferase reporter assay. HEK293T cells $\left(2.5 \times 10^{4}\right.$ cells/well) were seeded in a 24-well plate and cotransfected with $0.3 \mu \mathrm{g}$ of either the control vector or various miRNA constructs in conjunction with $0.1 \mu \mathrm{g}$ of the ET-1-3'UTR reporter construct (pmiR-RB-REPORT ${ }^{\mathrm{TM}}$; RiboBio) by using the Lipofectamine 2000 reagent (Invitrogen). After 48 h, the luciferase activity was measured using the Dual-Glo Luciferase Assay System kit (Promega, Madison, WI, USA). Luciferase readings were corrected for background, and firefly luciferase values were normalized to Renilla values to control for transfection efficiency. All of the assays were performed in triplicate in 3 independent experiments.

Methylation status of CpG islands. The genomic DNA of 5 gastric cancer cell lines, and normal and tumor tissue pairs from 50 gastric cancer patients were subjected to bisulfite conversion using the EZ DNA Methylation-Gold kit (Zymo Research Corporation, Orange, CA, USA). The bisulfite conversion reaction was conducted at $98^{\circ} \mathrm{C}$ for $10 \mathrm{~min}$, and then at $64^{\circ} \mathrm{C}$ for $2.5 \mathrm{~h}$, with a final incubation at $4^{\circ} \mathrm{C}$ for up to $20 \mathrm{~h}$ in a PCR thermocycler. Then, the modified genomic DNA was used for the methylation analysis of $\mathrm{CpG} 25$ and $\mathrm{CpG} 81$ of the mir-1-1 upstream using the specific methylation primers. The PCR conditions were as follows: $94^{\circ} \mathrm{C}$ for $10 \mathrm{~min}$, followed by 35 cycles of $94^{\circ} \mathrm{C}$ for $1 \mathrm{~min}, 60^{\circ} \mathrm{C}$ for $1 \mathrm{~min}$, and $72^{\circ} \mathrm{C}$ for $30 \mathrm{sec}$, with a final extension at $72^{\circ} \mathrm{C}$ for $10 \mathrm{~min}$ using a PCR thermocycler and HotStart Taq DNA polymerase (Qiagen, Hilden, Germany). The methylation status of the genomic DNA of individual samples was also examined with the Bst $\mathrm{UI}$ digestion (New England Biolabs, Beverly, MA, USA). The digested PCR fragments were then separated on $2 \%$ agarose gel. Detailed information is provided in a previous study (29). The individual primers used in the present study were as follows: CpG25-F, 5'-GGAGGGGTAGGATAGTAGTTTG AGT-3' and CpG25-R, 5'-AAAAAAACCTAACCTAAAAAA CCAAAA-3'; CpG81-F, 5' - GG TGA T T T T GT T TAG T T TAT TAT TAT-3' and CpG81-R, 5'-ATCAAAATTCCTA CCTCCCAACTA-3'.

Stem-loop RT-PCR of miR-1. Total RNA $(2 \mu \mathrm{g})$ was reversetranscribed with miR-1-specific stem-loop RT primers using SuperScript III Reverse Transcriptase (Invitrogen). The reaction was performed under the following incubation conditions: $30 \mathrm{~min}$ at $16^{\circ} \mathrm{C}$, followed by $20^{\circ} \mathrm{C}$ for $30 \mathrm{sec}, 42^{\circ} \mathrm{C}$ for $30 \mathrm{sec}$ and $50^{\circ} \mathrm{C}$ for $1 \mathrm{sec}$, totaling 50 cycles. The enzyme was subsequently inactivated by incubation at $85^{\circ} \mathrm{C}$ for $5 \mathrm{~min}$. Afterward, cDNA was diluted 10X for subsequent real-time PCR analysis using the miR-1-GSF primer. Gene expression was detected using a SYBR-Green I assay (Applied Biosystems, Foster City, CA, USA). The U6 expression was used as the internal control, 
Table I. Primer sequences.

\begin{tabular}{|c|c|}
\hline Constructed primers & Sequences \\
\hline ET-1-3'UTR-F & 5'-CCGCTCGAGCAGACCTTCGGGGCCTGTCTGAAG-3' \\
\hline ET-1-3'UTR-R & 5'-GAATGCGGCCGCACAGTAAGGAAAAAAATATTTATTTTC-3' \\
\hline miR-1-1-F & 5'-TCTACCGGTGGACACCAGGCAGCAGTGGC-3' \\
\hline miR-1-1-R & 5'-TCTGAATTCACAATGCTGGCGGGGACACG-3' \\
\hline let-7b-F & 5'-TCTACCGGTGGGCCTCTGCCTGTGGAGGA-3' \\
\hline let-7b-R & 5'-TCTGAATTCTCACTGAGGTAGGGGGCGGC-3' \\
\hline let-7c-F & 5'-TCTACCGGTTGGCAGGTTAGATGGTCAGAAGACA-3' \\
\hline let-7c-R & 5'-ACCTTCTTGCACACAAATTGGCTCA-3' \\
\hline miR-33a-F & 5'-TCTACCGGTCCCATAGCCTCTGTAAGCCC-3' \\
\hline miR-33a-R & 5'-TCTGAATTCGCTAAGGACATGTTCCCCGT-3' \\
\hline miR-101-F & 5'-TCTACCGGTTGCCTCCTCACGTCTCCAACCA-3' \\
\hline miR-101-R & 5'-TCTGAATTCTGGCTGCACCAACAACTACCCC-3' \\
\hline miR-125a-F & 5'-TCTACCGGTTTCTGTCCTTGTCCCTGCATC-3' \\
\hline miR-125a-R & 5'-TCTGAATTCCCATCGTGTGGGTCTCAAGG-3' \\
\hline miR-125b-F & 5'-TCTACCGGTTGCGCCCCCAGATACTGCGT-3' \\
\hline miR-125b-R & 5'-TCTGAATTCGGGGCATGCTGGGACTTCAGC-3' \\
\hline miR-134-F & 5'-TCTACCGGTCGGGCCATGGACAATGCGCT-3' \\
\hline miR-134-R & 5'-TCTGAATTCAGGGGCTGCCAAGGCTGACT-3' \\
\hline miR-135a-F & 5'-TCTACCGGTTCTTGTTTCCCGGTCCTTGT-3' \\
\hline miR-135a-R & 5'-TCTGAATTCACCCGACTGGTGGGCTATTA-3' \\
\hline miR-135b-F & 5'-TCTACCGGTTTTATGGCCAGGAAGCCACC-3' \\
\hline miR-135b-R & 5'-TCTGAATTCCAGCACACCCTGAAGGTCTC-3' \\
\hline miR-141-F & 5'-TCTACCGGTGGACACAATGGGCCCCAGCC-3' \\
\hline miR-141-R & 5'-TCTGAATTCAAAGCAGACGTCGCAGCCCC-3' \\
\hline miR-144-F & 5'-TCTACCGGTACACTGGCCCTGGGTCCCTA-3' \\
\hline miR-144-R & 5'-TCTGAATTCTGCCCTGGCAGTCAGTAGGTT-3' \\
\hline miR-203-F & 5'-TCTACCGGTGTCGGGGGCTCCTCTCTCCG-3' \\
\hline miR-203-R & 5'-TCTGAATTCCACCGCCAGTTCCTCGCTGG-3' \\
\hline miR-224-F & 5'-TCTACCGGTGCAGAGGGCTGGGCTACCTT-3' \\
\hline miR-224-R & 5'-TCTGAATTCCCCAGGGCCCAACTGGAAGAGT-3' \\
\hline miR-340-F & 5'-TCTACCGGTTCCAGCTTGAGTCTTCAAGAG-3' \\
\hline miR-340-R & 5'-TCTGAATTCAGAGTTGTGATCAGTAAATTAGA-3' \\
\hline miR-379-F & 5'-TCTACCGGTGCCTGCTTCCAATGCCAAAT-3' \\
\hline miR-379-R & 5'-TCTGAATTCGCCCAAGTTGCATCACTTCC-3' \\
\hline miR-410-F & 5'-TCTACCGGTGCCCTTTTGAGGGTAGGAGC-3' \\
\hline miR-410-R & 5'-TCTGAATTCAATGATTCAATGGCGGGGGT-3' \\
\hline miR-425-F & 5'-TCTACCGGTGTGCCCCTGACCCCCAGACA-3' \\
\hline miR-425-R & 5'-TCTGAATTCAGCAGGGAAACCCAGGGGCA-3' \\
\hline miR-494-F & 5'-TCTACCGGTACCGTCAGGAAAGCTCCAAT-3' \\
\hline miR-494-R & 5'-TCTGAATTCTCAGGAACAGGAAGTGCCTC-3' \\
\hline miR-495-F & 5'-TCTACCGGTCGCCTCTGCTCAGTGTCAGCC-3' \\
\hline miR-495-R & 5'-TCTGAATTCTCAGGGTCCCGTCGGGGATG-3' \\
\hline miR-590-F & 5'-TCTACCGGTGAACGTCAGCACCCTCCCCCCA-3' \\
\hline miR-590-R & 5'-TCTGAATTCTTGAGCGCCAGTGGCCAAGC-3' \\
\hline miR-873-F & 5'-TCTACCGGTGTGACCAGTGTCTGGGATGC-3' \\
\hline miR-873-R & 5'-TCTGAATTCCCTTGGTGGGATTCAACACCT-3' \\
\hline \multicolumn{2}{|l|}{ Gene-specific primer } \\
\hline miR-1-RT & 5'-CTCAACTGGTGTCGTGGAGTCGGCAATTCAGTTGAGATACATAC-3 \\
\hline miR-1-GSF & 5'-CGGCGGTGGAATGTAAAGAAGT-3' \\
\hline
\end{tabular}



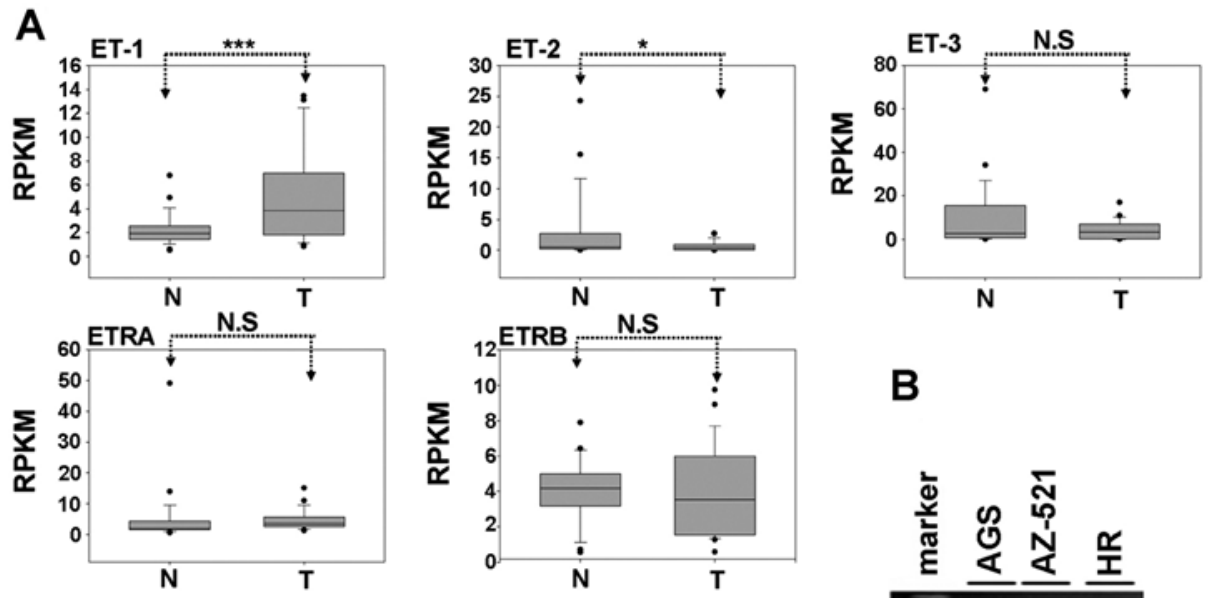

B
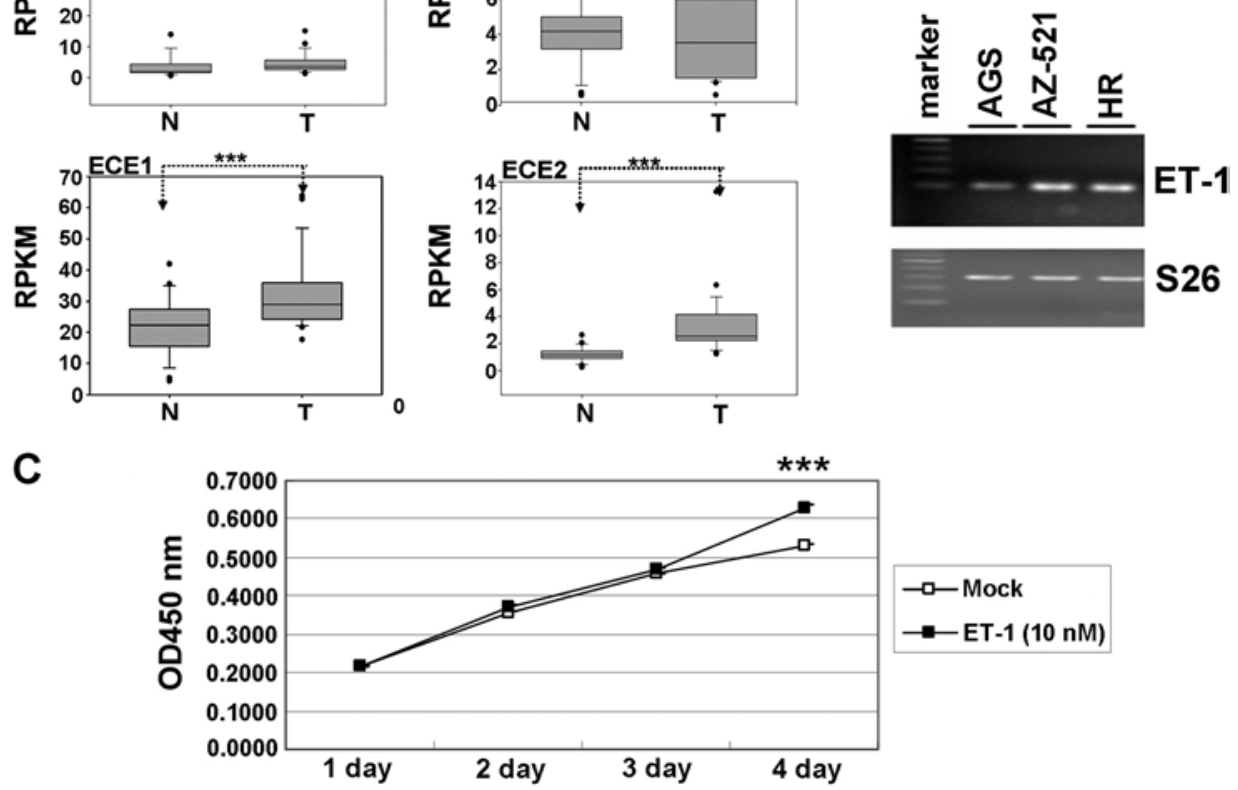

Figure 1. Expression levels and function of the endothelin axis in gastric cancer cell lines. (A) The expression levels of ET-1, ET-2, ET-3, ETRA, ETRB, ECE-1 and ECE-2 were examined between gastric cancer and adjacent normal from 29 patients by analyzing the TCGA database. The expression levels of the genes were presented in reads per kilobase per million reads (RPKM). The expression level between the tumor and normal cells was evaluated by conducting paired t-tests $\left(\mathrm{P}<0.05\right.$ was considered significant; $\mathrm{NS}$, non-significant; $\left.{ }^{*} \mathrm{P}<0.05,{ }^{* *} \mathrm{P}<0.01,{ }^{* * *} \mathrm{P}<0.001\right)$. (B) The mRNA level of ET-1 was assessed in 3 human gastric cancer cell lines. (C) Exogenous ET-1 stimulation enhanced AGS cell proliferation. ET-1, endothelin-1; ETRA and ETRB, G-protein-coupled receptors; ECE-1 and ECE-2, ET-converting enzymes; TCGA, The Cancer Genome Atlas.

and the expression levels of miR-1 were normalized to that of $\mathrm{U} 6\left(\Delta \mathrm{Ct}=\mathrm{Ct}_{\mathrm{miR-1}}-\mathrm{Ct}_{\mathrm{U} 6}\right)$.

Ectopic expression of miRNA candidates. Stable or transient gastric cell cultures expressing miR-1 candidates were generated using the transfected gastric cells with pLKO-pre-miRNA by Lipofectamine 2000. After 24 h of transfection, the stably expressed miRNA cells were generated by puromycin selection for 14 days. The shLuc targeting the luciferase gene provided puromycin resistance as the control. The expression levels of miRNAs were confirmed using real-time PCR.

Expression levels of ET axis genes and miRNAs were analyzed using The Cancer Genome Atlas (TCGA) data. The TCGA project collects RNA transcriptome and small RNA transcriptome data, which are obtained using the next-generation sequencing approach from various types of human cancers. For the present study, we downloaded all level-3 expression data for gastric cancer from the TCGA data portal (https://tcga-data.nci.nih.gov/tcga/dataAccessMatrix.htm). We used 29 tumor samples and 29 normal samples to analyze the expression levels of each miRNA and ET axis-related gene.
Cell proliferation assay. For cell proliferation analysis, we seeded 3,000 living AGS cells or AGS cells stably expressing miR-1 in 96-well plates. After exogenous ET-1 stimulation, we determined cell growth at 0, 1, 2, 3 and 4 days using WST-1 (Roche, Mannhein, Germany). All experiments were repeated 3 times.

\section{Results}

ET-1 is upregulated in gastric cancer. We first assessed the expression pattern of the ET axis genes (ET-1, ET-2, ET-2, ETRA, ETRB, ECE-1 and ECE-2) in gastric cancer by analyzing data from the database of TCGA. We obtained RNA transcriptome data on cancer tissues and the corresponding adjacent normal tissues from information on 29 gastric cancer patients. As shown in Fig. 1A, the expression levels of ET-1, ECE-1 and ECE-2 were significantly increased in gastric cancer compared to corresponding normal tissues $(\mathrm{P}<0.001)$. Conversely, ET-2 expression was decreased significantly in gastric cancer $(\mathrm{P}<0.05)$. We further examined the expression levels of ET-1 in 3 gastric cancer cell lines using the RT-PCR approach. As shown in Fig. 1B, we detected a greater abun- 


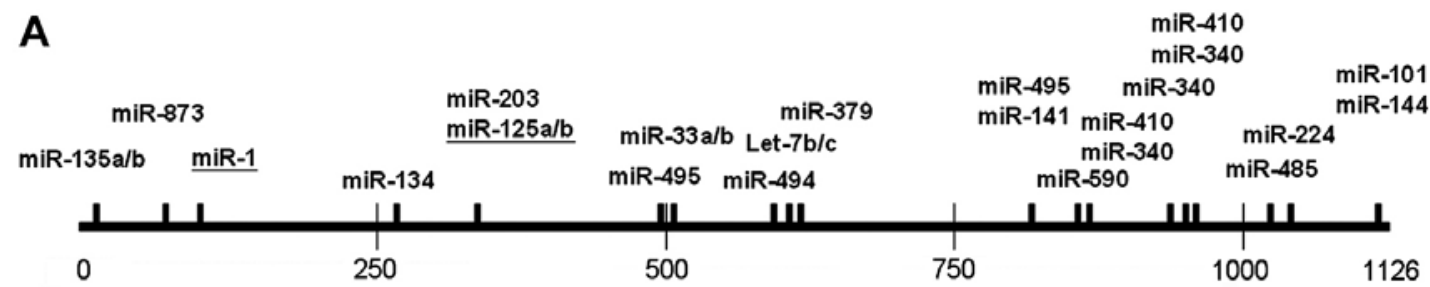

B

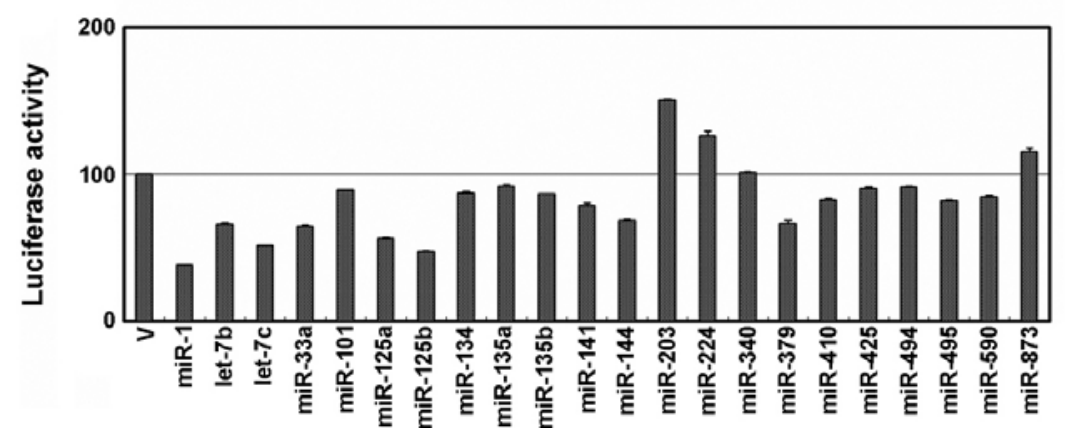

Figure 2. Putative microRNA candidates mediate ET-1 expression by targeting the 3'UTR of ET-1 in gastric cancer. (A) Schematic diagram of the luciferase constructs containing 3'UTR sequences of ET-1 (1,126 bp), which were cloned downstream of the luciferase gene. Predicted miRNA target sites within the 3'UTR of ET-1 through microRNA.org prediction program. The underlined miRNAs indicate that these candidates have been reported to target the 3'UTR sequence of ET-1. (B) ET-1 3'UTR was cloned into the pmiR-RB-Report vector and then were cotransfected with various miRNA constructs into HEK293T cells. Luciferase assay was conducted after 48 h cotransfection in HEK293T cells using the Dual-Glo Luciferase Reporter Assay System kit. ET-1, endothelin-1; 3'UTR, 3' untranslated region.

dance of ET-1 RNA in the AZ-521 and HR cells, and low expression in AGS cells. To investigate the putative function of ET-1 on gastric cancer, we further treated AGS cells with ET-1 (10 nM) for 4 days. We evaluated the potential influence of ET-1 on gastric cancer cell proliferation through WST-1 for $96 \mathrm{~h}$. As shown in Fig. 1C, cell proliferation in the ET-1 treatment groups was significantly increased compared with that in the control groups. These results demonstrate that ET-1 plays an oncogenic role in promoting the proliferation of gastric cancer cells. However, the mechanism that leads to ET-1 dysregulation in gastric cancer remains unclear. To further investigate the underlying mechanisms of ET-1 dysfunction in gastric cancer, we focused on the post-transcriptional regulation of ET-1 expression by miRNAs during the progression of gastric cancer.

Discovery of putative miRNAs that regulate ET-1 genes. Using a prediction program available on microRNA.org, we predicted that several miRNA candidates could suppress ET-1 expression by targeting its 3'UTR sequence. Among them, we selected 22 miRNA candidates, and constructed their pre-miRNA sequence into a pKLO expression vector (Fig. 2A). In addition, we cloned the full length of the 3'UTR (i.e., 1,126 bp) of ET-1 into the pmiR-RB-Report vector. Due to the fact that the HEK293T cells have a high transfection efficiency, we conducted a luciferase assay using HEK293T cells. As shown in Fig. 2B, miR-1, let-7c and miR-125b substantially inhibited luciferase activity by $>50 \%$ compared to the control group after transfection for $48 \mathrm{~h}$. Fifteen miRNA candidates slightly suppressed the luciferase activity, including let-7b, miR-33a, miR-101, miR-125a, miR-134, miR-135a/b, miR-141, miR-144, miR-379, miR-410, miR-425, miR-494, miR-495 and miR-590. Consistent with previous studies, our data indicated that miR-1 and miR-125a/b both suppressed ET-1 expression through post-transcriptional regulation. After using a reporter assay, we found that 18 miRNA candidates may be involved in ET-1 post-translational regulation through the targeting of its 3'UTR sequence.

Expression level of miRNA candidates from the TCGA database. After completing the reporter assay, we selected 18 miRNA candidates to further examine their expression levels in gastric cancer by analyzing data from the TCGA database. As shown in Fig. 3A, we identified 6 miRNAs (miR134, miR-135b, miR-141, miR-425, miR-494 and miR-590) and 5 miRNAs (miR-1, let-7c, miR-101, miR-125a and miR-144) that were significantly upregulated and downregulated, respectively, in gastric cancer. Since the expression level of ET-1 was upregulated in the gastric cancer cells compared with the corresponding normal cells, miRNA candidate expression should decrease in gastric cancer. According to their expression levels in gastric cancer, we selected 6 miRNAs (miR-1, miR-101, miR-125a/b, miR-144 and let-7c) for further confirmation in the AGS cells. These miRNA candidates were ectopically expressed in the AGS cells, and let-7c, miR-1, miR-101, miR-125a/b and miR-144 were found to significantly suppress luciferase activity (Fig. 3B). In addition, we found that the endogenous protein levels of ET-1 were inhibited by let-7c, miR-1, miR-101, miR-125a and miR-144 overexpression (Fig. 3C).

DNA hypermethylation silences miR-1 expression in gastric cancer. Among the listed miRNAs, we selected miR-1 to further examine the biological function and the regulatory mechanisms in gastric cancer. The expression of miR-1 originates from 2 paralogous genes, mir-1-1 and mir-1-2, located on chromosomes 20 and 18, respectively. As shown in Fig. 4A, $4 \mathrm{CpG}$ islands are located at the putative transcription start 

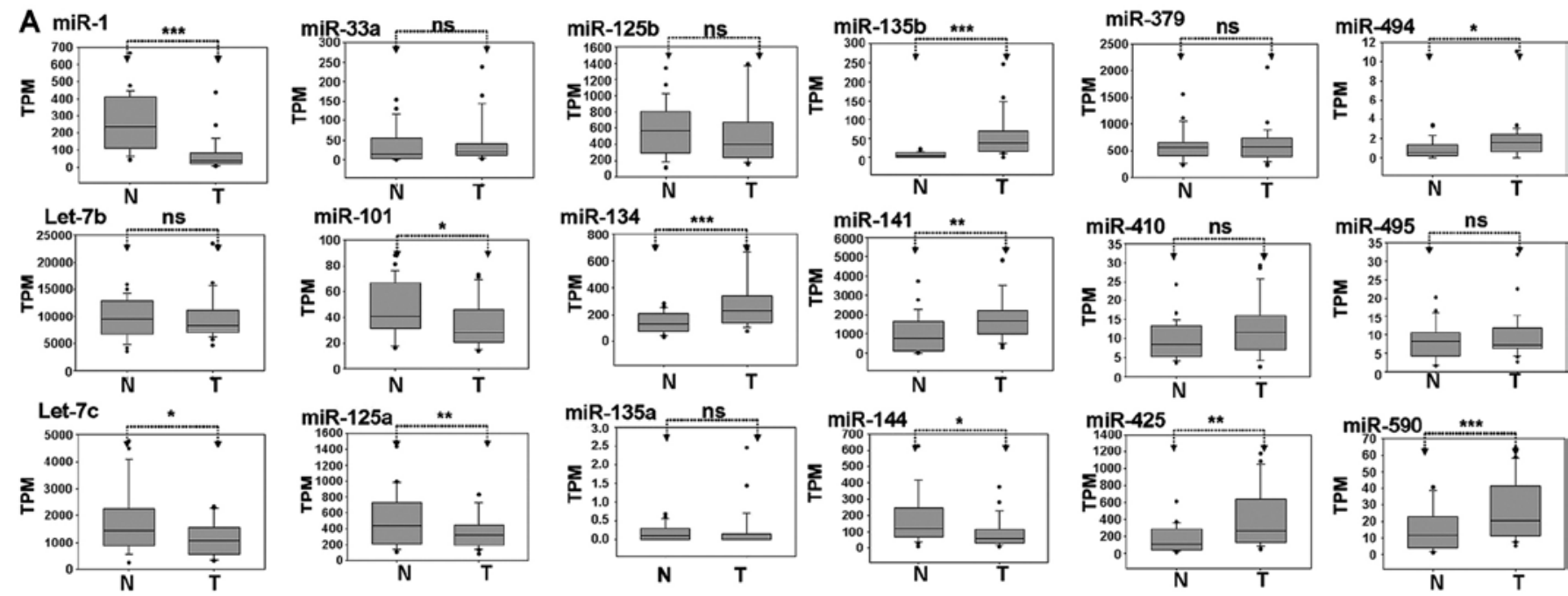

B
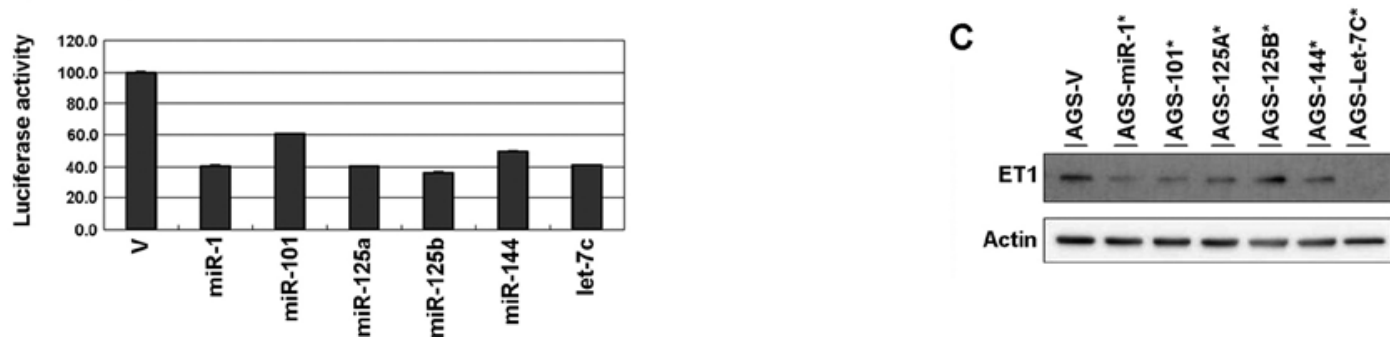

Figure 3. Suppression of ET-1 expression by miRNAs in gastric cancer cells. (A) Expression levels of the miRNA candidates in gastric cancer. Comparison of the miRNA expression levels between the tumor $(\mathrm{T})$ and corresponding normal $(\mathrm{N})$ tissues obtained from 29 gastric cancer patients, which were analyzed using the TCGA dataset. The expression levels of the miRNAs are presented in transcripts per million (TPM). The expression level between tumor and normal cells was evaluated by conducting paired t-tests $\left(\mathrm{P}<0.05\right.$ was considered significant; NS, non-significant, $\left.{ }^{*} \mathrm{P}<0.05,{ }^{* *} \mathrm{P}<0.01,{ }^{* * *} \mathrm{P}<0.001\right)$. (B) The reporter of ET-1 3'UTR was cotransfected with various miRNA constructs [vector (V), miR-1, miR-101, miR-125a/b, miR-144 and let-7c] into AGS cells. Luciferase assay was conducted after $48 \mathrm{~h}$ of cotransfection in AGS cells using the Dual-Glo Luciferase Reporter Assay System kit. (C) After $48 \mathrm{~h}$ transfection, the endogenous protein levels of ET-1 were examined by western blot analysis. ET-1, endothelin-1; 3'UTR, 3' untranslated region.

sites of the miR-1-1 loci, according to the UCSC database. We analyzed the methylation statuses of the CpG25 and CpG81 regions in 5 human gastric cancer cell lines by using a COBRA approach. As shown in Fig. 4B, the CpG81 regions were completely methylated in the AGS and AZ-521 cells, yet unmethylated in the HR cells. After treatment for 4 days with 5-Aza-dC, the expression levels of miR-1 were restored in the AGS, AZ-521 and HR cells (Fig. 4C). These data indicate that DNA methylation contributes to the modulation of miR-1 transcriptional activity in gastric cancer cell lines.

By analyzing TCGA data, we found that the expression levels of miR-1 were significantly decreased in the gastric cancer cells compared with the corresponding adjacent normal cells (Fig. 3A). Therefore, we further examined the methylation status of the CpG81 regions in 50 primary gastric cancers and the corresponding adjacent control, and observed a high frequence of DNA hypermethylation in gastric cancer (26 of 50). These results indicated that DNA hypermethylation leads to low expression levels of miR-1 in gastric cancer (Fig. 4D).

miR-1 suppresses gastric cancer cell growth. The ectopic expression of miR-1 in AGS cells increased mature miR-1 expression by $\sim 250$-fold (Fig. 5A). The growth of AGS cells with miR-1 overexpression was substantially inhibited compared with those transfected with the vector (Fig. 5B). Furthermore, the clonogenic survival of AGS cells also decreased after ectopic expression of miR-1 (Fig. 5C). Overall, our results imply that ET-1 overexpression may be due to the silencing of miR-1 expression by DNA hypermethylation in gastric cancer. In addition, we identified several miRNA candidates that could modulate the ET-1 oncogenic function, and could also act as potential therapeutic targets for therapy in the future.

\section{Discussion}

Dysfunction of the endothelin (ET) axis is known to be a feature of human cancer, including lung, prostate, colorectal, liver, breast, ovarian and oral cancer as well as melanomas. Abnormal activation of the ET axis leads to promotion of cell growth, metastasis and angiogenesis, and suppression of apoptosis (4-11). By analyzing TCGA data, we found that the expression levels of ET-1, ECE-1 and ECE-2 were significant increased in the gastric cancer compared to the corresponding normal tissues (Fig. 1). A previous study reported that the plasma big-ET-1 level in gastric cancer was significantly higher than that in the control (30). These findings imply that the expression level of ET-1 is increased in gastric cancer. Although ECE-1 (31) and ECE-2 were found to be upregulated in a number of cancers, including prostate, ovarian and oral cancer, thyroid carcinoma, and breast cancer (32-35), the expression of ECE-1 and ECE-2 was not reported in gastric 
A

chr20:61,146,694-61,154,784

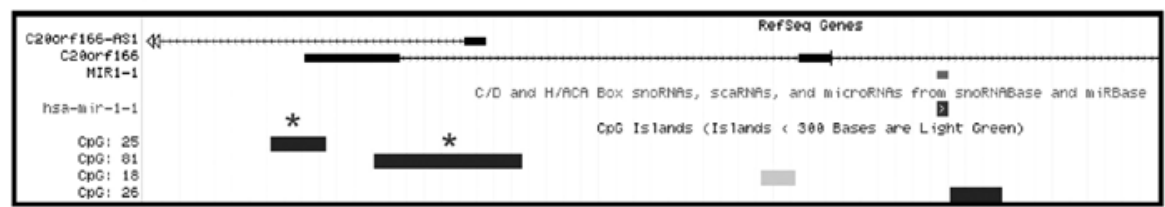

B

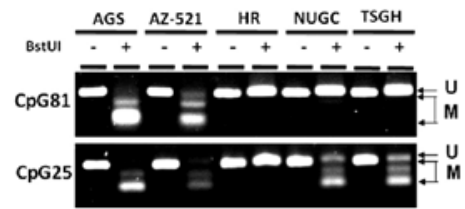

D

C
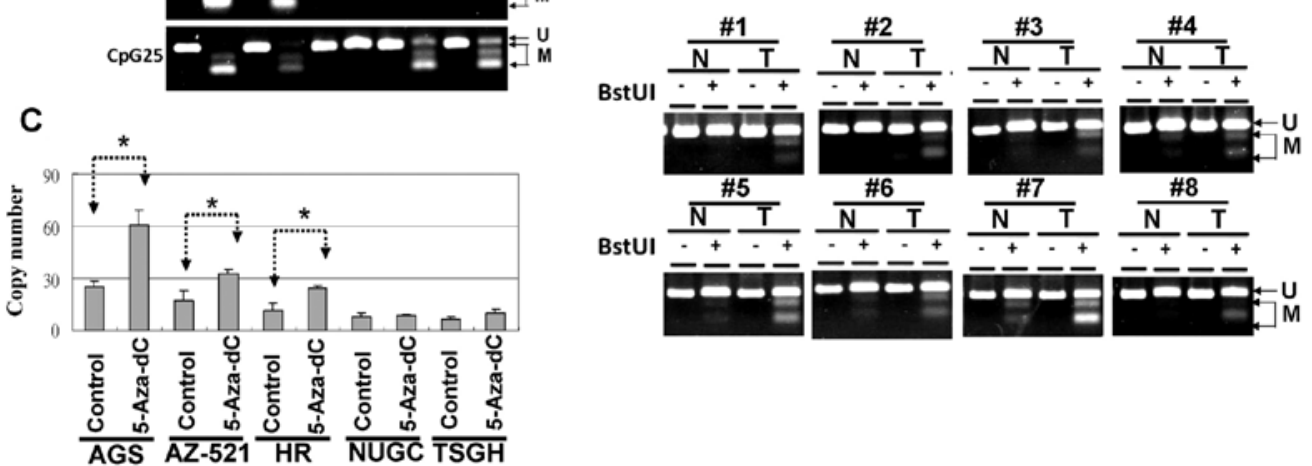

Figure 4. Expression of miR-1-1 is regulated by DNA methylation in gastric cancer. (A) Schematic representation of the locations of the mir-1-1 genes Expressed sequence tag (EST) transcripts were identified from the UCSC website (http://genome.ucsc.edu/). Asterisks indicate the analyzed CpG islands (CpG25 and CpG81). (B) DNA methylation status of CpG25 and CpG81 of the mir-1-1 upstream region in 5 gastric cancer cell lines was examined by COBRA approach. $\mathrm{M}$ and $\mathrm{U}$ indicate the presence of methylated $\mathrm{CpGs}$ and unmethylated CpGs, respectively. (C) Real-time PCR analysis of miR-1 in 5 gastric cancer cell lines before and after treatment with $10 \mu \mathrm{M} 5$-Aza-dC; U6 expression was used as an internal control. Gene expression was calculated relative to that of an internal control $(\Delta \mathrm{Ct})$. The number of copies of miR-1 was calculated using the standard equation $10,000 \times\left(2^{-\Delta C}\right)$. (D) DNA methylation status of CpG81 of the mir-1-1 upstream region in gastric cancers examined by COBRA approach in normal $(\mathrm{N})$ and tumor $(\mathrm{T})$ tissue pairs from 50 gastric cancer patients. Eight representative cases are presented. $\mathrm{M}$ and $\mathrm{U}$ indicate the presence of methylated and unmethylated CpGs, respectively.

A

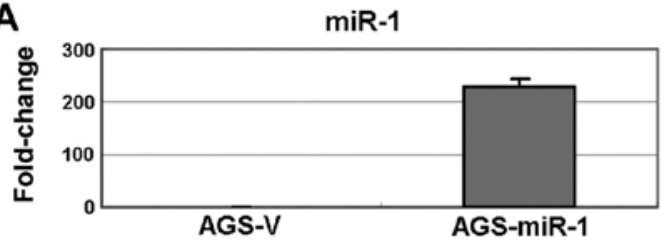

B

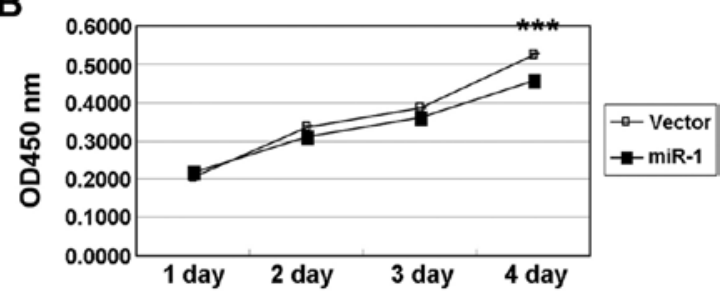

C

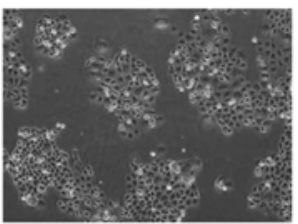

Vector

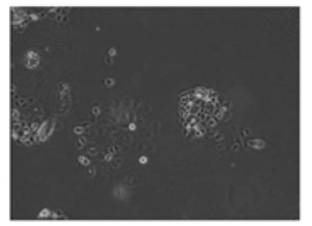

miR-1
Figure 5. Ectopically overexpressed miR-1 inhibits cell growth. (A) Overexpression of miR-1 by Lipofectamine 2000 transfected into AGS cells and the expression level of miR-1 were examined by stem-loop RT-PCR. (B and C) Overexpression of miR-1 suppressed cell proliferation in AGS cells. Cells were transfected with either control pLKO-shLuc (as control) or pLKO-miR-1 for $24 \mathrm{~h}$, which was followed by puromycin selection. Cell proliferation was examined by WST-1, and the colony formation was observed by microscopy. cancer. Previous studies found that, in human cancer, ET-2 and ET-3 expression levels are silenced by DNA hypermethylation of their promoter regions $(36,37)$. In the present study, we observed that the expression level of ET-2 was significant decreased in gastric cancer. However, what mechanism is involved in the loss of expression in gastric cancer remains unclear. These studies support our finding that the ET-1 axis is overexpressed, and that ET-2 is downregulated in gastric cancer.

In the past decade, miRNAs have emerged as playing important roles via modulation of the cell cycle, cell growth and cell motility in gastric cancer progression $(21,22)$. In the present study, we identified 15 miRNAs that may modulate ET-1 expression by targeting 3'UTR. Among them, 6 miRNAs (miR-134, miR-135b, miR-141, miR-425, miR-494 and miR-590) and 5 miRNAs (miR-1, let-7c, miR-101, miR$125 \mathrm{a}$ and miR-144) showed significant upregulation and downregulation, respectively, in gastric cancer. Consistent with our findings, previous studies have revealed that miR-135b and miR-425 act as oncomiRs involved in the tumorigenesis of gastric cancer (38-40). Furthermore, miR-125a/b, miR-144 and miR-101 have been reported to be significantly decreased and display a tumor-suppressive function by silencing their oncongenic targets in gastric cancer. Here, we demonstrated that miR-125a/b, miR-101 and miR-144 suppressed ET1 expression by targeting the $3^{\prime} \mathrm{UTR}$ in gastric cancer. In addition, previous studies have reported that miR-1, miR-125a/b, miR-155 and miR-199 suppress ET-1 expression by targeting 3'UTR (23-28). In numerous cancers, miR-1 performs a 
tumor-suppressive function by suppressing tumor cell growth and migration in prostate cancer, hepatocellular, bladder and esophageal squamous cell carcinoma, and lung cancer $(29,41-$ 48). Furthermore, hypermethylation of the promoter region of mir-1-1 resulted in low expression in hepatocellular carcinoma and colorectal cancer $(29,48-50)$. In the present study, we also demonstrated that miR-1 expression levels were suppressed by DNA methylation and that ectopic miR-1 expression led to the suppression of gastric cancer cell growth. Collectively, our results imply that ET-1 overexpression may be due to DNA hypermethylation resulting in the silencing of miR-1 expression in gastric cancer.

In solid tumors, the ET-axis pathway plays a critical oncogenic role in facilitating cancer growth and metastasis. Various investigations have demonstrated that the ET-1-ETRA axis is a potential therapeutic target for cancer therapy. To date, several drugs have been designed as ETRA antagonists for anticancer growth and metastasis. The ET-1 is a short intercellular peptide derived from numerous tumor cells, and has emerged as a potential therapeutic target or biomarker for human cancer. In the present study, our identification of the post-translational regulation of ET-1 may lead to novel insights into the function of ET-1 in gastric cancer, and may provide a potential therapeutic target.

\section{Acknowledgements}

This study was supported by grants from the Kaohsiung Veterans General Hospital (VGHKS 102-020, VGHKS 102-074, VGHKS 103-066 and VGHKS 103-108).

\section{References}

1. Yanagisawa M, Kurihara H, Kimura S, et al: A novel potent vasoconstrictor peptide produced by vascular endothelial cells. Nature 332: 411-415, 1988.

2. Kedzierski RM and Yanagisawa M: Endothelin system: the double-edged sword in health and disease. Annu Rev Pharmacol Toxicol 41: 851-876, 2001.

3. Rubanyi GM and Polokoff MA: Endothelins: molecular biology, biochemistry, pharmacology, physiology, and pathophysiology. Pharmacol Rev 46: 325-415, 1994.

4. Grimshaw MJ: Endothelins in breast tumour cell invasion. Cancer Lett 222: 129-138, 2005.

5. Asham E, Shankar A, Loizidou M, et al: Increased endothelin-1 in colorectal cancer and reduction of tumour growth by $\mathrm{ET}_{\mathrm{A}}$ receptor antagonism. Br J Cancer 85: 1759-1763, 2001.

6. Eberle J, Fecker LF, Orfanos CE and Geilen CC: Endothelin-1 decreases basic apoptotic rates in human melanoma cell lines. J Invest Dermatol 119: 549-555, 2002.

7. Douglas ML, Richardson MM and Nicol DL: Endothelin axis expression is markedly different in the two main subtypes of renal cell carcinoma. Cancer 100: 2118-2124, 2004.

8. Kim TH, Xiong H, Zhang Z and Ren B: $\beta$-Catenin activates the growth factor endothelin-1 in colon cancer cells. Oncogene 24: 597-604, 2005.

9. Abdel-Gawad IA, Hassanein HM, Bahgat NA, et al: Study of endothelin-1 and vascular endothelial grow th factor in patients with cancer colon. J Egypt Natl Canc Inst 20: 216-223, 2008.

10. Said N, Smith S, Sanchez-Carbayo M and Theodorescu D: Tumor endothelin-1 enhances metastatic colonization of the lung in mouse xenograft models of bladder cancer. J Clin Invest 121 : 132-147, 2011.

11. Nelson JB, Udan MS, Guruli G and Pflug BR: Endothelin-1 inhibits apoptosis in prostate cancer. Neoplasia 7: 631-637, 2005.

12. Rosano L, Spinella F and Bagnato A: Endothelin 1 in cancer: biological implications and therapeutic opportunities. Nat Rev Cancer 13: 637-651, 2013.
13. Nelson JB, Lee WH, Nguyen SH, et al: Methylation of the 5' CpG island of the endothelin $\mathrm{B}$ receptor gene is common in human prostate cancer. Cancer Res 57: 35-37, 1997.

14. Pao MM, Tsutsumi M, Liang G, Uzvolgyi E, Gonzales FA and Jones PA: The endothelin receptor $\mathrm{B}(E D N R B)$ promoter displays heterogeneous, site specific methylation patterns in normal and tumor cells. Hum Mol Genet 10: 903-910, 2001.

15. Cohen AJ, Belinsky S, Franklin W and Beard S: Molecular and physiologic evidence for $5^{\prime} \mathrm{CpG}$ island methylation of the endothelin B receptor gene in lung cancer. Chest 121: 27S-28S, 2002.

16. Lo KW, Tsang YS, Kwong J, To KF, Teo PM and Huang DP: Promoter hypermethylation of the $E D N R B$ gene in nasopharyngeal carcinoma. Int J Cancer 98: 651-655, 2002.

17. Zhao BJ, Sun DG, Zhang M, Tan SN and Ma X: Identification of aberrant promoter methylation of $E D N R B$ gene in esophageal squamous cell carcinoma. Dis Esophagus 22: 55-61, 2009.

18. Mathieu MN and Chevillard C: Endothelin-1 and ETA receptor subtype are expressed in the gastric HGT-1 cell line. J Cardiovasc Pharmacol 26 (Suppl 3): S508-S509, 1995.

19. Ferrari-Bravo A, Franciosi C, Lissoni P, Fumagalli L and Uggeri F: Effects of oncological surgery on endothelin-1 secretion in patients with operable gastric cancer. Int J Biol Markers 15: 56-57, 2000.

20. Stow LR, Jacobs ME, Wingo CS and Cain BD: Endothelin-1 gene regulation. FASEB J 25: 16-28, 2011

21. Pan HW, Li SC and Tsai KW: MicroRNA dysregulation in gastric cancer. Curr Pharm Des 19: 1273-1284, 2013.

22. Wu HH, Lin WC and Tsai KW: Advances in molecular biomarkers for gastric cancer: miRNAs as emerging novel cancer markers. Expert Rev Mol Med 16: e1, 2014.

23. Jacobs ME, Wingo CS and Cain BD: An emerging role for microRNA in the regulation of endothelin-1. Front Physiol 4: 22, 2013.

24. Yeligar S, Tsukamoto $H$ and Kalra VK: Ethanol-induced expression of ET-1 and ET-BR in liver sinusoidal endothelial cells and human endothelial cells involves hypoxia-inducible factor-1 $\alpha$ and microRNA-199. J Immunol 183: 5232-5243, 2009.

25. Li D, Yang P, Xiong Q, et al: MicroRNA-125a/b-5p inhibits endothelin-1 expression in vascular endothelial cells. J Hypertens 28 : 1646-1654, 2010.

26. Li D, Yang P, Li H, et al: MicroRNA-1 inhibits proliferation of hepatocarcinoma cells by targeting endothelin-1. Life Sci 91: 440-447, 2012.

27. Li D, He B, Zhang H, et al: The inhibitory effect of miRNA-1 on ET-1 gene expression. FEBS Lett 586: 1014-1021, 2012.

28. Feng B, Cao Y, Chen S, Ruiz M and Chakrabarti S: miRNA-1 regulates endothelin-1 in diabetes. Life Sci 98: 18-23, 2014.

29. Chen WS, Leung CM, Pan HW, et al: Silencing of miR-1-1 and miR-133a-2 cluster expression by DNA hypermethylation in colorectal cancer. Oncol Rep 28: 1069-1076, 2012.

30. Teng XJ, Shen ZX, Xiang JJ, et al: Pre- and post-operative plasma big endothelin-1 levels in patients with gastric carcinoma undergoing radical gastrectomy. Anticancer Res 26: 2503-2507, 2006.

31. Whyteside AR, Hinsley EE, Lambert LA, McDermott PJ and Turner AJ: ECE-1 influences prostate cancer cell invasion via ET-1-mediated FAK phosphorylation and ET-1-independent mechanisms. Can J Physiol Pharmacol 88: 850-854, 2010.

32. Smollich M, Götte M, Yip GW, et al: On the role of endothelinconverting enzyme-1 (ECE-1) and neprilysin in human breast cancer. Breast Cancer Res Treat 106: 361-369, 2007.

33. Dawson LA, Maitland NJ, Berry P, Turner AJ and Usmani BA: Expression and localization of endothelin-converting enzyme-1 in human prostate cancer. Exp Biol Med 231: 1106-1110, 2006.

34. Awano S, Dawson LA, Hunter AR, Turner AJ and Usmani BA: Endothelin system in oral squamous carcinoma cells: specific siRNA targeting of ECE-1 blocks cell proliferation. Int J Cancer 118: 1645-1652, 2006.

35. Van Beneden R, Michel L, Havaux X, Delos M and Donckier J: Increased expression of endothelin-1 converting enzyme in human thyroid carcinoma. Clin Endocrinol 60: 146-147, 2004.

36. Wiesmann F, Veeck J, Galm O, et al: Frequent loss of endothelin-3 (EDN3) expression due to epigenetic inactivation in human breast cancer. Breast Cancer Res 11: R34, 2009.

37. Wang R, Lohr CV, Fischer K, et al: Epigenetic inactivation of endothelin-2 and endothelin-3 in colon cancer. Int J Cancer 132: 1004-1012, 2013.

38. Lim JY, Yoon SO, Seol SY, et al: Overexpression of miR-196b and HOXA10 characterize a poor-prognosis gastric cancer subtype. World J Gastroenterol 19: 7078-7088, 2013. 
39. Ma J,Liu J, Wang Z, et al: NF-kappaB-dependent microRNA-425 upregulation promotes gastric cancer cell growth by targeting PTEN upon IL-1 $\beta$ induction. Mol Cancer 13: 40, 2014.

40. Peng WZ, Ma R, Wang F, Yu J and Liu ZB: Role of miR-191/425 cluster in tumorigenesis and diagnosis of gastric cancer. Int J Mol Sci 15: 4031-4048, 2014.

41. Yoshino H, Chiyomaru T, Enokida H, et al: The tumoursuppressive function of $m i R-1$ and $m i R-133 a$ targeting TAGLN2 in bladder cancer. Br J Cancer 104: 808-818, 2011.

42. Wu CD, Kuo YS, Wu HC and Lin CT: MicroRNA-1 induces apoptosis by targeting prothymosin alpha in nasopharyngeal carcinoma cells. J Biomed Sci 18: 80, 2011.

43. Wang F, Song G, Liu M, Li X and Tang H: miRNA-1 targets fibronectin 1 and suppresses the migration and invasion of the HEp2 laryngeal squamous carcinoma cell line. FEBS Lett 585: 3263-3269, 2011.

44. Nohata N, Sone Y, Hanazawa T, et al: miR-1 as a tumor suppressive microRNA targeting TAGLN2 in head and neck squamous cell carcinoma. Oncotarget 2: 29-42, 2011.

45. Nasser MW, Datta J, Nuovo G, et al: Down-regulation of micro-RNA-1 (miR-1) in lung cancer. Suppression of tumorigenic property of lung cancer cells and their sensitization to doxorubicin-induced apoptosis by miR-1. J Biol Chem 283: 33394-33405, 2008.
46. Kinoshita T, Nohata N, Fuse M, et al: Tumor suppressive microRNA-133a regulates novel targets: moesin contributes to cancer cell proliferation and invasion in head and neck squamous cell carcinoma. Biochem Biophys Res Commun 418: 378-383, 2012.

47. Hudson RS, Yi M, Esposito D, et al: MicroRNA-1 is a candidate tumor suppressor and prognostic marker in human prostate cancer. Nucleic Acids Res 40: 3689-3703, 2012.

48. Datta J, Kutay H, Nasser MW, et al: Methylation mediated silencing of microRNA-1 gene and its role in hepatocellular carcinogenesis. Cancer Res 68: 5049-5058, 2008.

49. Migliore C, Martin V, Leoni VP, et al: MiR-1 downregulation cooperates with MACC1 in promoting $M E T$ overexpression in human colon cancer. Clin Cancer Res 18: 737-747, 2012.

50. Suzuki H, Takatsuka S, Akashi H, et al: Genome-wide profiling of chromatin signatures reveals epigenetic regulation of microRNA genes in colorectal cancer. Cancer Res 71: 56465658,2011 